Supporting Information for

\title{
Realization of a High-voltage and High-rate Nickel-rich NCM Cathode Material for LIBs by Co and Ti Dual- Modification
}

Xiaoyu Zhang, Yuegang Qiu, Fangyuan Cheng, Peng Wei, Yuyu Li, Yi Liu, Shixiong

Sun, Yue Xu, Qing Li, Chun Fang*, Jiantao Han* and Yunhui Huang

State Key Laboratory of Material Processing and Die \& Mould Technology, School of Materials Science and Engineering, Huazhong University of Science and Technology,

Wuhan, Hubei 430074, China

Corresponding author

* E-mail addresses: fangchun@hust.edu.cn (C. Fang), jthan@hust.edu.cn (J. Han) 


\section{Supporting Figures}

Table S1. Crystal structure parameters of NCM-811, T1-NCM, T1Co0.5-NCM.

\begin{tabular}{llll}
\hline Samples & NCM811 & T1-NCM & T1Co0.5-NCM \\
\hline $\mathrm{a}(\AA)$ & 2.870591 & 2.872250 & 2.872445 \\
$\mathrm{~b}(\AA)$ & 2.870591 & 2.872250 & 2.872445 \\
$\mathrm{c}(\AA)$ & 14.196160 & 14.207430 & 14.207973 \\
$\mathrm{c} / \mathrm{a}$ & 4.9454 & 4.9464 & 4.9463 \\
$\mathrm{I}(003) / \mathrm{I}(104)$ & 1.16 & 1.21 & 1.29 \\
$\mathrm{Ni}$ in Li site $(\%)$ & 3.41 & 3.1 & 3.04 \\
\hline
\end{tabular}


Table S2. The comparison between our result and reported results.

\begin{tabular}{|c|c|c|c|c|c|c|}
\hline Compounds & $\begin{array}{l}\text { Modified } \\
\text { strategy }\end{array}$ & $\begin{array}{l}\text { Voltage } \\
\text { range } \\
\text { (V) }\end{array}$ & $\begin{array}{l}\text { Discharge } \\
\text { capacity } \\
\left(\mathrm{mAh} \mathrm{g}^{-1}\right)\end{array}$ & $\begin{array}{l}\text { Current } \\
\text { density }\end{array}$ & $\begin{array}{c}\text { Capacity } \\
\text { rentention (25 } \\
\left.{ }^{\circ} \mathrm{C}\right)\end{array}$ & $\begin{array}{r}\text { Refere } \\
\text { nce }\end{array}$ \\
\hline $\begin{array}{c}\mathrm{LiNi}_{0.85} \mathrm{Co}_{0.1} \mathrm{Mn}_{0.05} \\
\mathrm{O}_{2}\end{array}$ & $\begin{array}{l}\text { PEDOT } \\
\text { coating }\end{array}$ & $\begin{array}{l}2.7- \\
4.3\end{array}$ & 178 & $1 \mathrm{C}$ & $91 \% / 100^{\text {th }}$ & {$[1]$} \\
\hline $\begin{array}{c}\mathrm{LiNi}_{0.76} \mathrm{Mn}_{0.14} \mathrm{Co}_{0.1} \\
\mathrm{O}_{2}\end{array}$ & $\begin{array}{l}\mathrm{Li}_{3} \mathrm{PO}_{4} \\
\text { coating }\end{array}$ & $\begin{array}{c}2.7- \\
4.5\end{array}$ & 212 & $\mathrm{C} / 3$ & $91.6 \% / 200^{\text {th }}$ & {$[2]$} \\
\hline $\begin{array}{c}\mathrm{LiNi}_{0.8} \mathrm{Mn}_{0.1} \mathrm{Co}_{0.1} \mathrm{O} \\
2\end{array}$ & $\begin{array}{c}\mathrm{V}_{2} \mathrm{O}_{5} \\
\text { coating }\end{array}$ & $\begin{array}{l}2.7- \\
4.3\end{array}$ & 170 & $2 \mathrm{C}$ & $76.4 \% / 300^{\text {th }}$ & [3] \\
\hline $\begin{array}{c}\mathrm{LiNi}_{0.81} \mathrm{Co}_{0.1} \mathrm{Mn}_{0.19} \\
\mathrm{O}_{2}\end{array}$ & $\begin{array}{l}\mathrm{Li}_{\mathrm{x}} \mathrm{CoO}_{2} \\
\text { coating }\end{array}$ & $\begin{array}{c}3.0- \\
4.3\end{array}$ & 200 & $1 \mathrm{C}$ & $80 \% / 300^{\text {th }}$ & [4] \\
\hline $\begin{array}{c}\mathrm{LiNi}_{0.815} \mathrm{Co}_{0.15} \mathrm{Al}_{0.03} \\
{ }_{5} \mathrm{O}_{2}\end{array}$ & $\begin{array}{l}\mathrm{Li}_{2} \mathrm{MoO}_{4} \\
\text { coating }\end{array}$ & $\begin{array}{c}2.7- \\
4.3\end{array}$ & 162.5 & $1 \mathrm{C}$ & $90 \% / 300^{\text {th }}$ & {$[5]$} \\
\hline $\mathrm{LiNi}_{0.8} \mathrm{Co}_{0.1} \mathrm{Mn}_{0.1} \mathrm{O}$ & $\begin{array}{l}\text { PANI } \\
\text { coating }\end{array}$ & $\begin{array}{c}2.8- \\
4.3\end{array}$ & 200 & $\begin{array}{c}200 \\
\mathrm{~mA} / \mathrm{g}\end{array}$ & $88.7 \% / 100^{\text {th }}$ & [6] \\
\hline $\begin{array}{c}\mathrm{LiNi}_{0.82} \mathrm{Co}_{0.12} \mathrm{Mn}_{0.06} \\
\mathrm{O}_{2}\end{array}$ & $\begin{array}{c}\mathrm{Ge}^{3+} \\
\text { doping }\end{array}$ & $\begin{array}{c}2.0- \\
4.5\end{array}$ & 210 & $0.1 \mathrm{C}$ & $81 \% / 100^{\text {th }}$ & [7] \\
\hline $\begin{array}{c}\mathrm{LiNi}_{0.9} \mathrm{Co}_{0.05} \mathrm{Mn}_{0.05} \\
\mathrm{O}_{2}\end{array}$ & B doping & $\begin{array}{c}2.7- \\
4.3\end{array}$ & 210 & $0.5 \mathrm{C}$ & $91 \% / 100^{\text {th }}$ & [8] \\
\hline $\mathrm{LiNi}_{0.8} \mathrm{Co}_{0.1} \mathrm{Mn}_{0.1} \mathrm{O}$ & Al doping & $\begin{array}{c}2.7- \\
4.5\end{array}$ & 200 & $0.33 \mathrm{C}$ & $87.7 \% / 250^{\text {th }}$ & [9] \\
\hline $\begin{array}{c}\mathrm{LiNi}_{0.6} \mathrm{Co}_{0.2} \mathrm{Mn}_{0.2} \mathrm{O} \\
2\end{array}$ & Ta doping & $\begin{array}{c}3.0- \\
4.5\end{array}$ & 180 & $1 \mathrm{C}$ & $83.6 \% / 100^{\text {th }}$ & {$[10]$} \\
\hline $\mathrm{LiNi}_{0.8} \mathrm{Co}_{0.2} \mathrm{O}_{2}$ & Ti doping & $\begin{array}{c}2.8- \\
4.5\end{array}$ & 195 & $1 \mathrm{C}$ & $\begin{array}{c}86.38 \% / 100^{\mathrm{t}} \\
\mathrm{h}\end{array}$ & [11] \\
\hline $\begin{array}{c}\mathrm{LiNi}_{0.8} \mathrm{Co}_{0.1} \mathrm{Mn}_{0.1} \mathrm{O} \\
2\end{array}$ & $\mathrm{Ti}$ and $\mathrm{La}$ & $\begin{array}{c}2.7- \\
4.3\end{array}$ & 177.52 & $1 \mathrm{C}$ & $89.2 \% / 200^{\text {th }}$ & {$[12]$} \\
\hline \multirow[t]{2}{*}{$\mathbf{O}_{2}$} & $\begin{array}{c}\text { Ti and Co } \\
\text { modificatio } \\
n\end{array}$ & $\begin{array}{c}2.7- \\
4.3\end{array}$ & $\begin{array}{c}(195.2) \\
179\end{array}$ & $\begin{array}{c}(0.2 \mathrm{C}) \\
1 \mathrm{C}\end{array}$ & $97.1 \% / 400^{\text {th }}$ & $\begin{array}{l}\text { This } \\
\text { work }\end{array}$ \\
\hline & & $\begin{array}{c}2.7- \\
4.5\end{array}$ & $\begin{array}{c}(218.7) \\
197.1\end{array}$ & $\begin{array}{c}(0.2 \mathrm{C}) \\
1 \mathrm{C}\end{array}$ & $92 \% / 400^{\text {th }}$ & $\begin{array}{l}\text { This } \\
\text { work }\end{array}$ \\
\hline
\end{tabular}


2.7 - (234.6) (0.2C) This

$4.7 \quad 206.1 \quad 1 \mathrm{C} \quad 74.2 \% / 400^{\text {th }}$ work

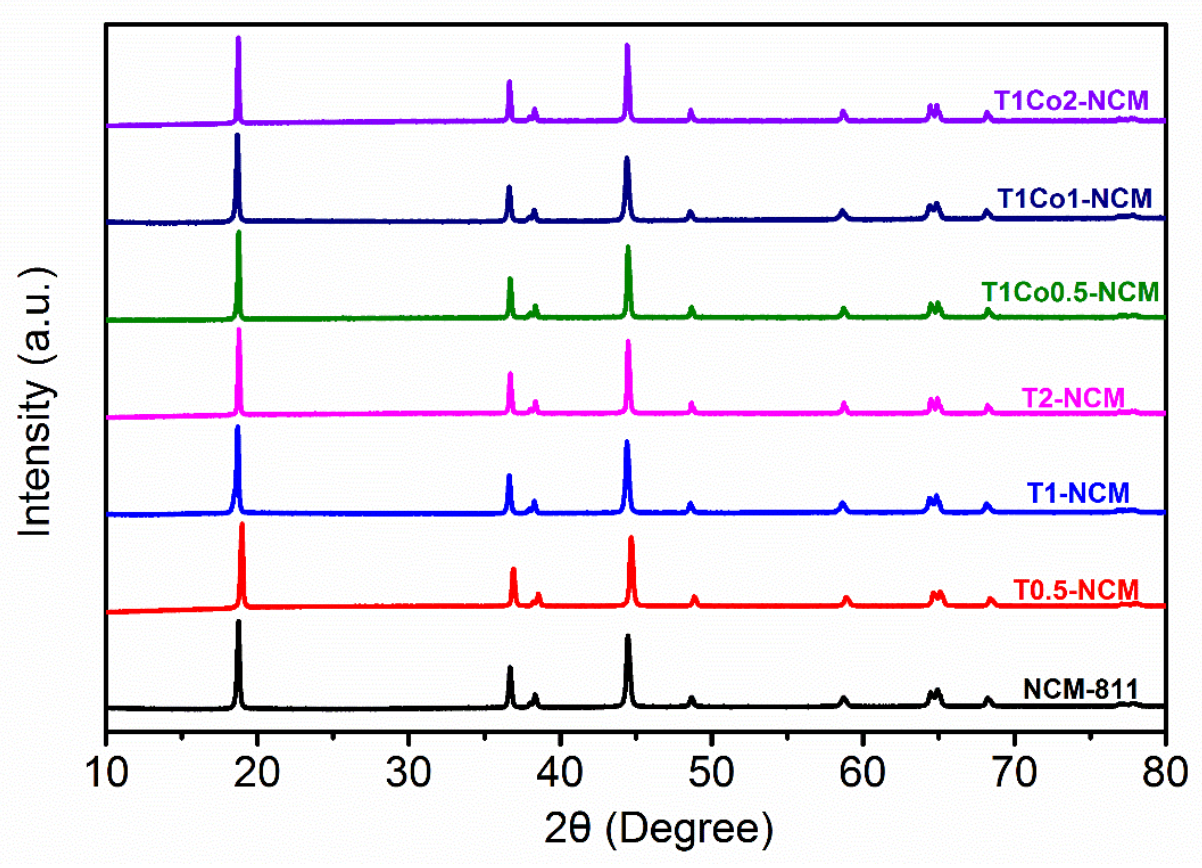

Figure S1 XRD patterns of different samples

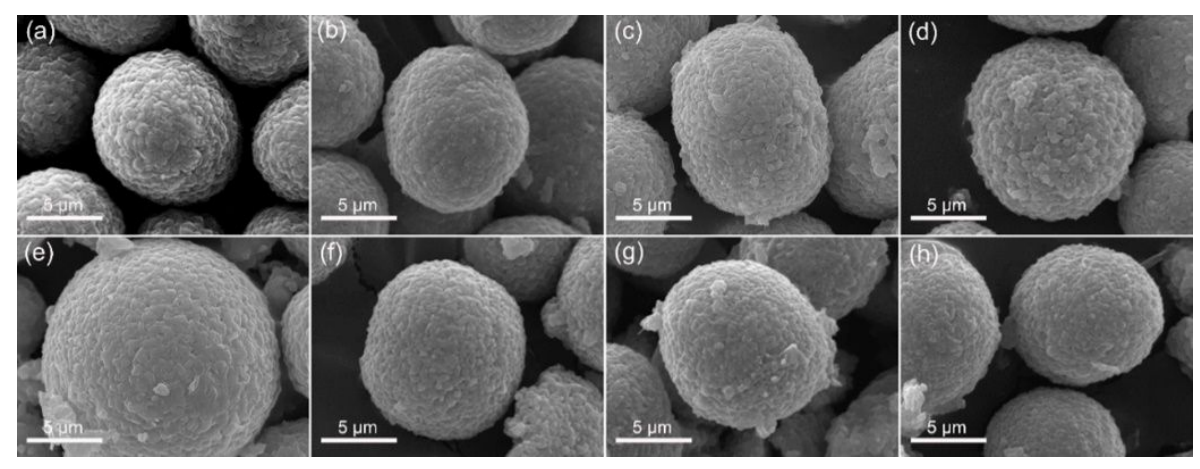

Figure S2. SEM for (a) NCM precursor, (b) NCM-811, (c) T0.5-NCM, (d) T1-NCM, (e) T2-NCM, (f) T1Co0.5-NCM (g) T1Co1-NCM (h) T1Co2-NCM. 


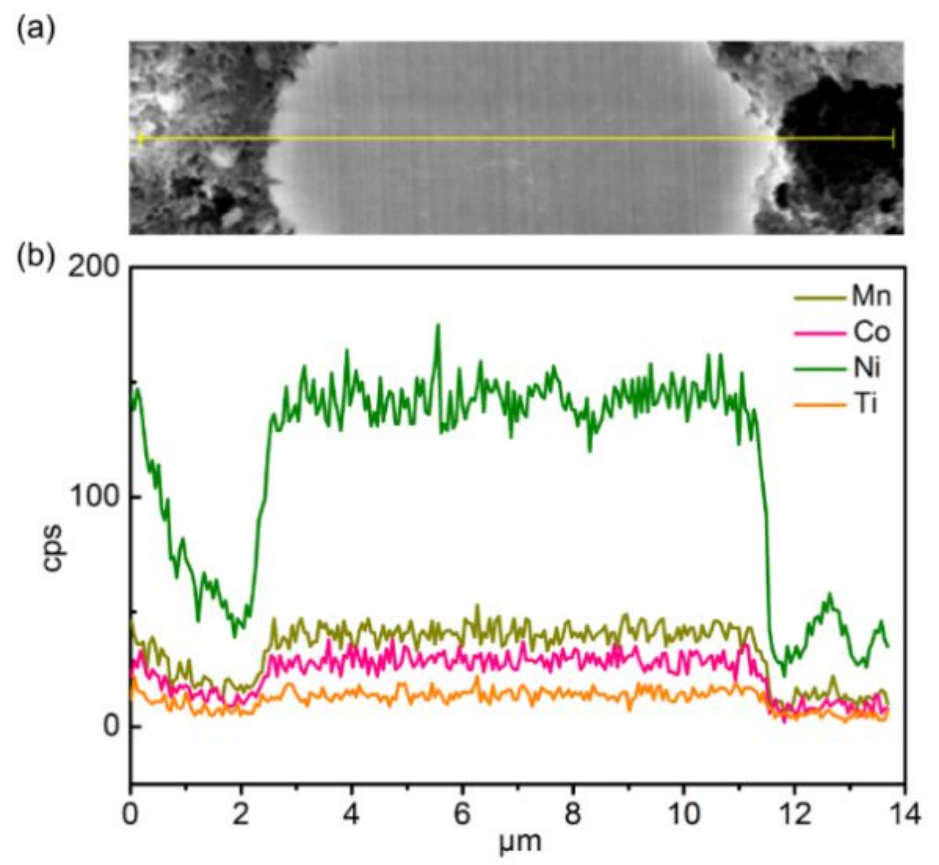

Figure S3. (a) Cross-sections of the T1Co0.5-NCM, (b) Linear scan of elements for T1Co0.5-NCM.
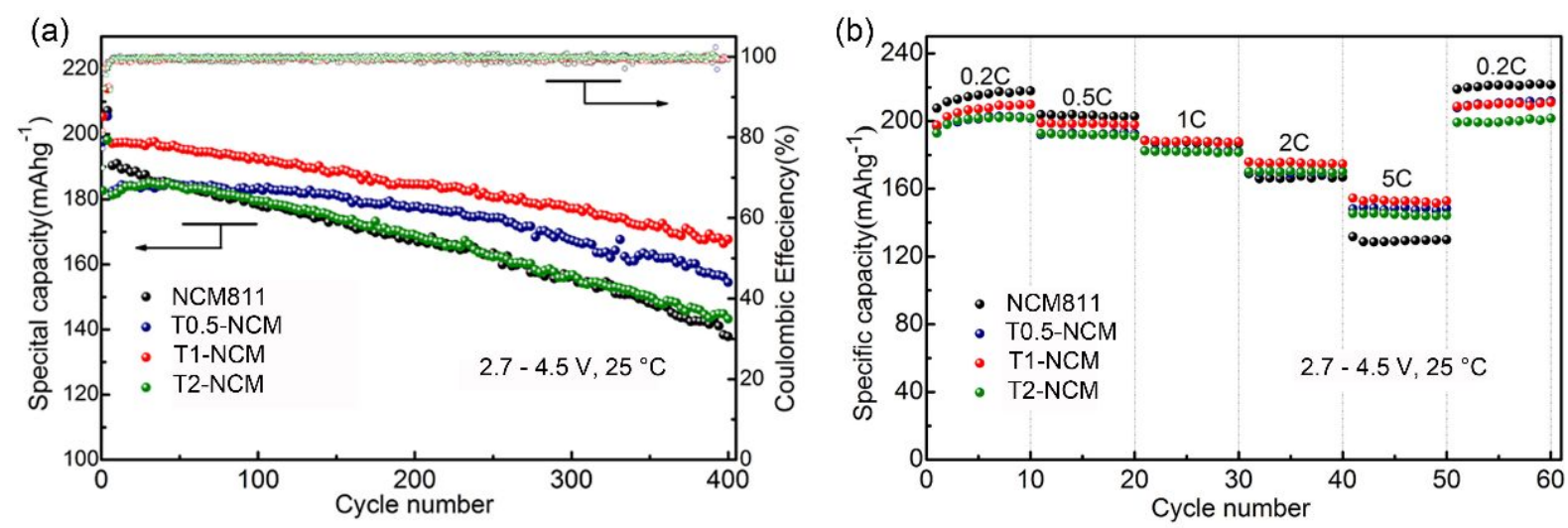

Figure S4. (a) cycle performance, (b) rate capability of different amount of Ti doping $(0.5 \%, 1 \%, 2 \%)$ between $2.7-4.5 \mathrm{~V}, 1 \mathrm{C}, 25^{\circ} \mathrm{C}$. 

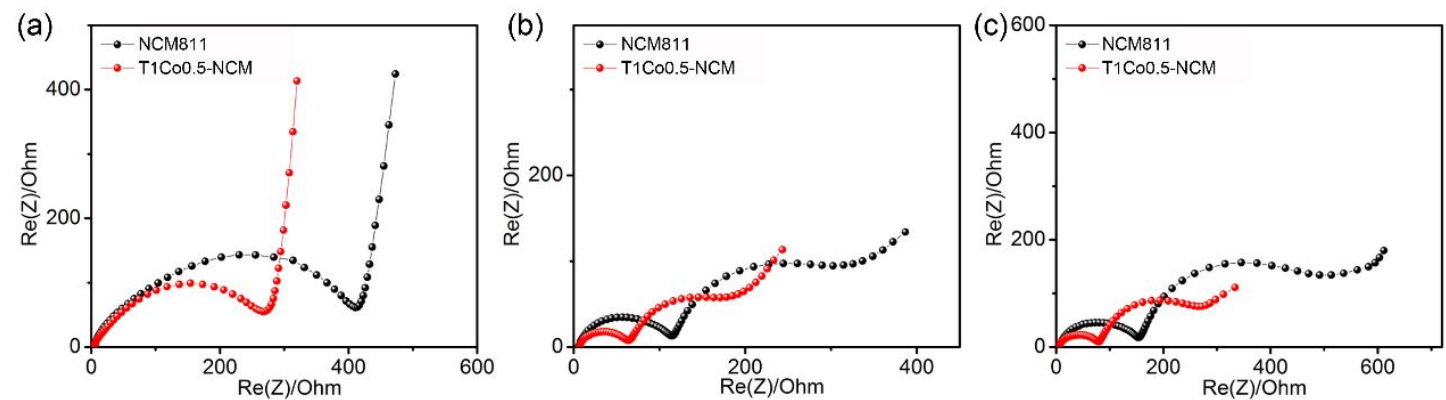

Figure S5. Nyquist plots of the electrochemical impedance of NCM-811 and

T1Co0.5-NCM after (a) 0, (b) 10 th, (c) 100 th cycles, measured at the fully charged state.
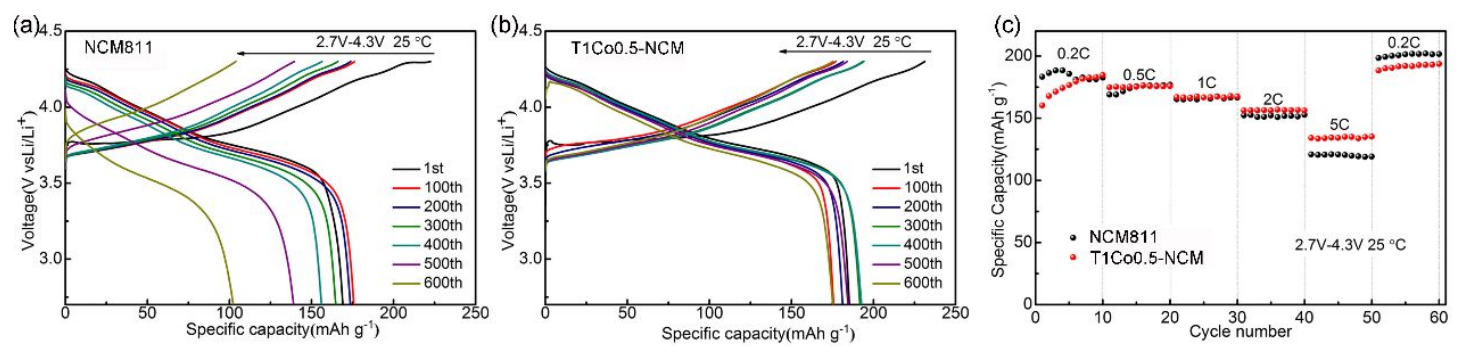

Figure S6. Typical charge-discharge profiles of (a) pristine NCM-811 and (b)

T1Co0.5-NCM between $2.7-4.3 \mathrm{~V}$ at $1 \mathrm{C}, 25^{\circ} \mathrm{C}$. (c) rate capability between $2.7-4.3$ $\mathrm{V}, 25^{\circ} \mathrm{C}$.
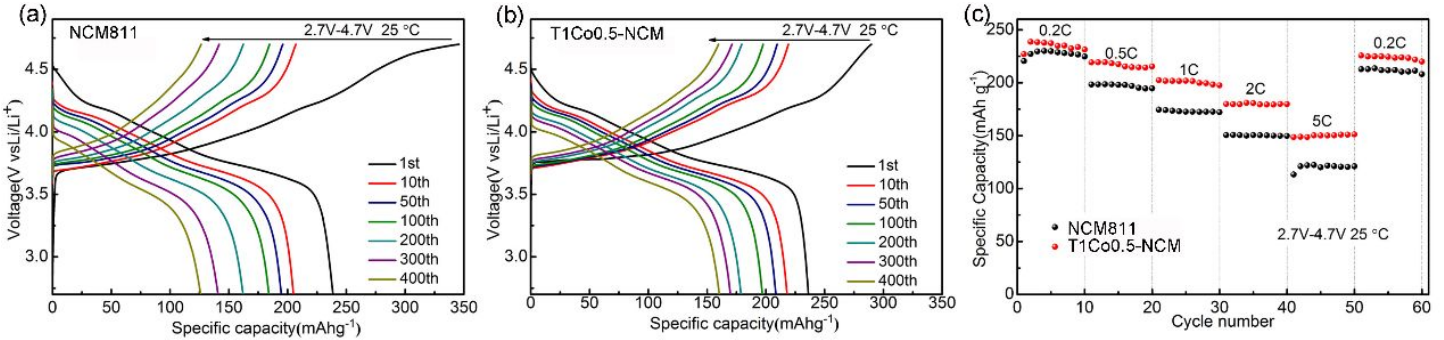

Figure S7. Typical charge-discharge profiles of (a) NCM-811, (b) T1Co0.5-NCM between $2.7-4.7 \mathrm{~V}$ at $1 \mathrm{C}, 25^{\circ} \mathrm{C}$. (c) rate capability between $2.7-4.7 \mathrm{~V}$ at $1 \mathrm{C}$, $25{ }^{\circ} \mathrm{C}$. 


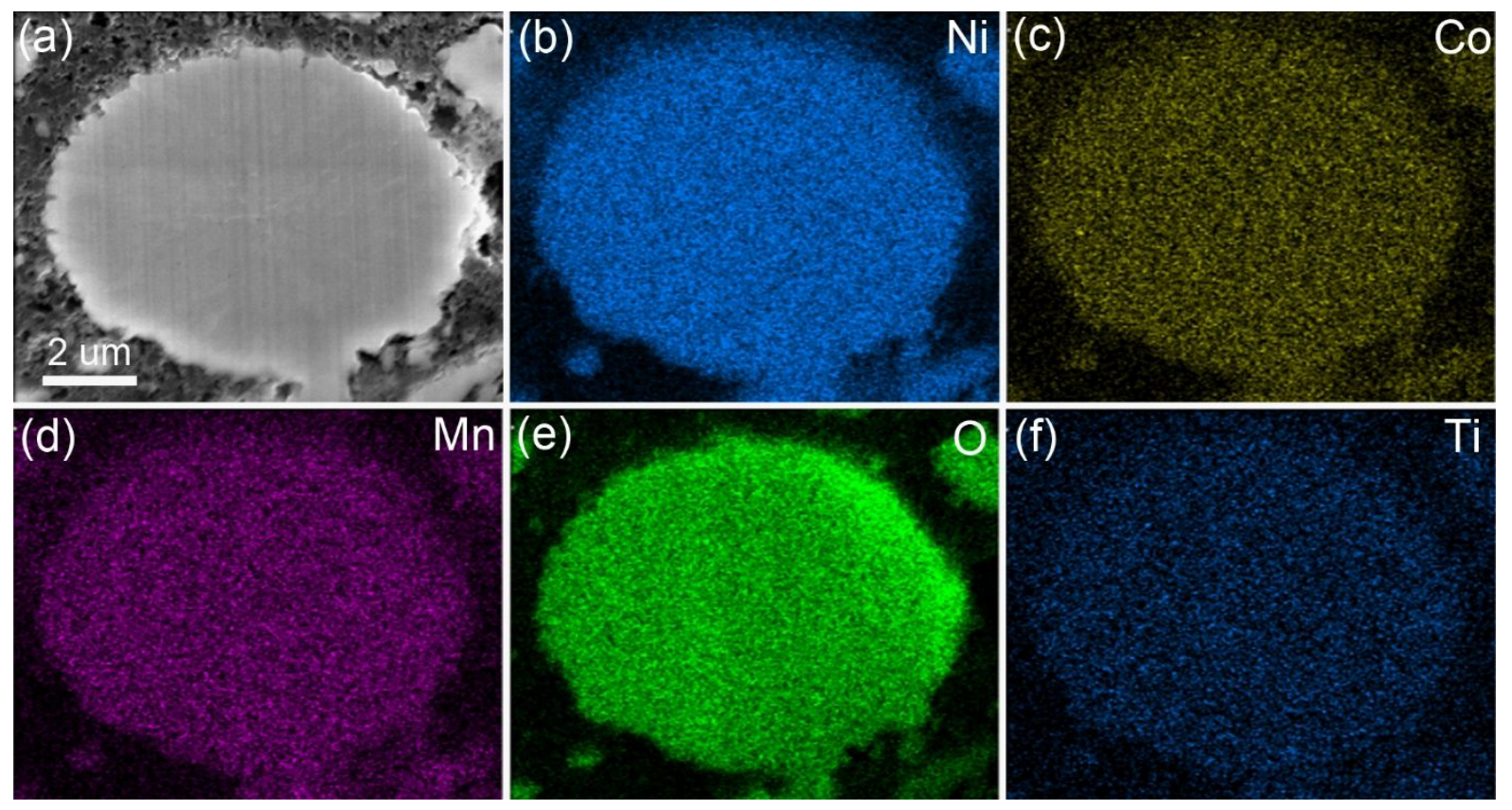

Figure S8. SEM and EDS-mapping for the cross-sections of the T1Co0.5-NCM sample (prepared by Focused Ion beam) after 400 cycles charging to $4.5 \mathrm{~V}$.

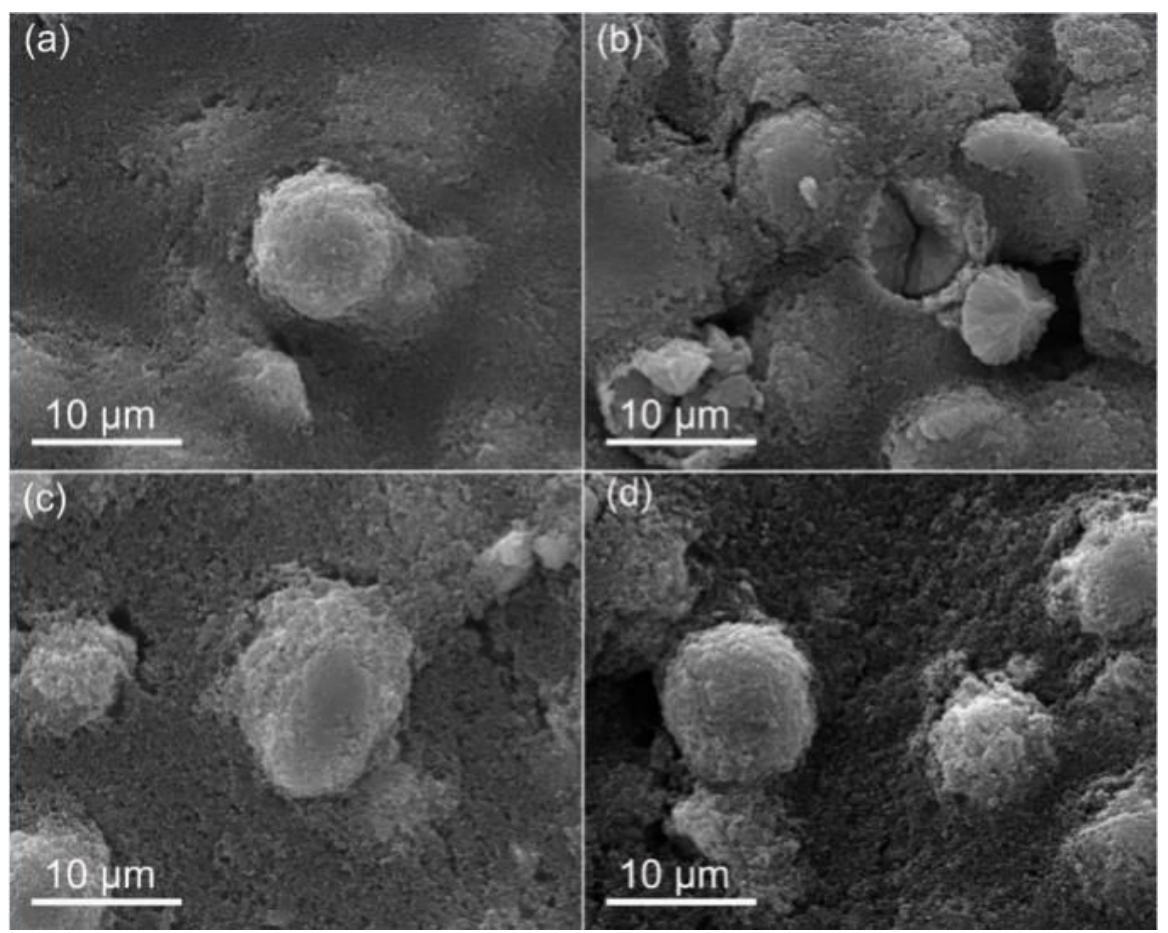


Figure S9. SEM images of NCM-811 and T1Co0.5-NCM electrodes (a, c) before the cycle, (b, d) after 400 cycles.
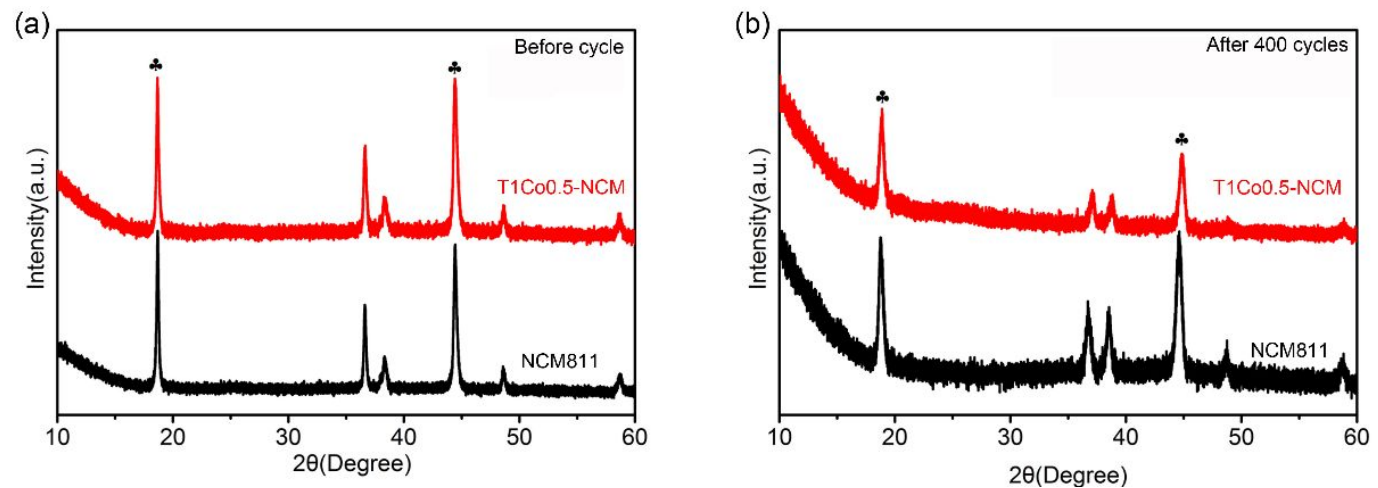

Figure S10. XRD patterns for cathode materials (a) before the cycle, (b) after 400 cycles. 
Reference:

[1] G.-L. Xu, Q. Liu, K. K. S. Lau, Y. Liu, X. Liu, H. Gao, X. Zhou, M. Zhuang, Y. Ren, J. Li, M. Shao, M. Ouyang, F. Pan, Z. Chen, K. Amine, G. Chen, Nat. Energy 2019, 6, 484.

[2] P. Yan, J. Zheng, J. Liu, B. Wang, X. Cheng, Y. Zhang, X. Sun, C. Wang, J.-G. Zhang, Nat. Energy 2018, 7, 600.

[3] X. Xiong, Z. Wang, H. Guo, Q. Zhang, X. Li, J. Mater. Chem. A 2013, 4, 1284.

[4] H. Kim, S. Lee, H. Cho, J. Kim, J. Lee, S. Park, S. H. Joo, S. H. Kim, Y. G. Cho, H. K. Song, S. K. Kwak, J. Cho, Adv. Mater. 2016, 23, 4705.

[5] C. Xu, W. Xiang, Z. Wu, Y. Xu, Y. Li, Y. Wang, Y. Xiao, X. Guo, B. Zhong, ACS Appl Mater Interfaces 2019, 18, 16629.

[6] Q. Gan, N. Qin, Y. Zhu, Z. Huang, F. Zhang, S. Gu, J. Xie, K. Zhang, L. Lu, Z. Lu, ACS Appl. Mater. Interfaces 2019, 13, 12594.

[7] M. Tang, J. Yang, N. Chen, S. Zhu, X. Wang, T. Wang, C. Zhang, Y. Xia, J. Mater. Chem. A 2019, 6080.

[8] K.-J. Park, H.-G. Jung, L.-Y. Kuo, P. Kaghazchi, C. S. Yoon, Y.-K. Sun, Adv. Energy Mater. 2018, 25, 1801202. 
[9] W. Zhao, L. Zou, H. Jia, J. Zheng, D. Wang, J. Song, C. Hong, R. Liu, W. Xu, Y. Yang, J. Xiao, C. Wang, J.-G. Zhang, ACS Appl. Energ. Mater. 2020, 4, 3369.

[10] B. Chu, S. Liu, L. You, D. Liu, T. Huang, Y. Li, A. Yu, ACS Sustainable Chemistry \& Engineering 2020, 8, 3082.

[11] D. Kong, J. Hu, Z. Chen, K. Song, C. Li, M. Weng, M. Li, R. Wang, T. Liu, J. Liu, M. Zhang, Y. Xiao, F. Pan, Adv. Energy Mater. 2019, 41, 1901756.

[12] H. Yang, H. H. Wu, M. Ge, L. Li, Y. Yuan, Q. Yao, J. Chen, L. Xia, J. Zheng, Z. Chen, J. Duan, K. Kisslinger, X. C. Zeng, W. K. Lee, Q. Zhang, J. Lu, Adv. Funct. Mater. 2019, 13, 1808825. 